\title{
Evolution of particle density in high-energy pp collisions
}

\author{
I. Bautista $^{\dagger *}$, C. Pajares *and J. Dias de Deus ${ }^{\dagger}$
}

August 27, 2018

\begin{abstract}
We study the evolution of the particle density, $d n / d \eta$ at fixed $\eta$, with the beam rapidity $Y$ in the framework of string percolation model. Our main results are: $(i)$ The width of the "plateau" increases proportionally to $Y,(i i)$ limiting fragmentation is violated, and ( $i i i)$ the particle density, reduces to a step function.
\end{abstract}

We work in the framework of the string percolation and one of the main results is the presence of an extended "plateau" with a length $\Delta y$ proportional to $Y(\Delta y \simeq 1.4 Y)$, where $Y$ is the beam rapidity in the center of mass system: $\left.Y \equiv \ln (\sqrt{(} s) / m_{p}\right)$. This result favors saturation models [1-4] with formation of longitudinal fields (flux tubes, effective strings) and naturally explains the presence of long range (pseudo-rapidity) correlations and the ridge phenomenon. See [5] for a discussion. Consequences of dominance of the "plateau" are the violation of limiting fragmentation and evolution towards a step function.

In the string percolation model multi-particle production in a two nuclei collision at high energy can be explained in terms of the produced strings along the collision axis, between the projectile and target. These strings decay into new ones by $q \bar{q}$ or $q q-\bar{q} \bar{q}$ pair production and subsequently hadronize to produce the observed hadrons. Due to confinement, the color of these

*IGFAE and Departamento de Física de Partículas, Univ. of Santiago de Compostela, 15782, Santiago de Compostela, Spain

†CENTRA, Departamento de Física, IST, Av. Rovisco Pais, 1049-001 Lisboa, Portugal 
strings is confined to a small area in transverse space $S_{1}=\pi r_{0}^{2}$, with $r_{0} \simeq 0.2-0.3 \mathrm{fm}$. With increasing energy and/or atomic number of the colliding particles, the number of strings $N_{s}$ grows and they start to overlap forming clusters, very much like disks in two-dimensional percolation theory. At a certain critical density, a macroscopic cluster appears, which marks the percolation phase transition [6] [7].

In string percolation the relevant quantity is the transverse impact parameter density $\eta^{t}$ which, in the case of pp collisions, we write as

$$
\eta^{t} \equiv\left(\frac{r_{0}}{R_{p}}\right)^{2} \bar{N}_{s}
$$

where $r_{0}$ is the single string transverse size, $R_{p} \simeq 1 \mathrm{fm}$ is the proton transverse size and $\bar{N}^{s}$ is the average number of single strings. For values of $\eta^{t}$ below the critical 2- dimensional density for percolation, $\eta_{c}^{t} \simeq 1.15-1.5$ [8], the formed strings do not interact and collective effects are not present. For values $\eta^{t} \gtrsim \eta_{c}^{t}$ one observes the formation of long strings due to fusion, stretching between the beam and the target. We assume that $\eta^{t} \gtrsim \eta_{c}^{t}$.

The particle density $d n / d y$ at mid-rapidity is related to the average number $\bar{N}_{s}$ of strings

$$
\frac{d n}{d y} \sim F\left(\eta^{t}\right) \bar{N}_{s}
$$

where $F\left(\eta^{t}\right)$ is the color reduction factor [9], due to color summation of random colors,

$$
F\left(\eta^{t}\right) \equiv \sqrt{\frac{1-e^{-\eta^{t}}}{\eta^{t}}} .
$$

For small $\eta^{t}, F\left(\eta^{t}\right) \rightarrow 1$, and for $\eta^{t} \gtrsim \eta_{c}^{t}, F\left(\eta^{t}\right) \rightarrow \frac{1}{\sqrt{\eta^{t}}}$. Starting with an exponential growth of the average number $\bar{N}_{s}$ of strings,

$$
\bar{N}_{s} \sim e^{2 \lambda Y}
$$

with $\lambda \simeq 0.2-0.3[10,11]$, we write for the particle density, at $y \simeq 0$,

$$
\frac{d n}{d y} \sim e^{\lambda Y}
$$

and for the full rapidity distribution, [12,13],

$$
\left.\frac{d n}{d y}\right|_{p p}=\left.a e^{\lambda Y} \frac{d n}{d y}\right|^{s}
$$


where $\left.\frac{d n}{d y}\right|^{s}$ is the single string density, $\left.\frac{d n}{d \eta}\right|_{p p}$ is the pp density, and $a$ is a constant, depending on the nature of produced particles.

Note that the exponential behavior in (4), $\bar{N}^{s} \sim e^{2 \lambda Y}$, is not an assumption but results from a simple application of conservation of energy to $d n / d y$ - making use of (2), (3) and the high density limit - to obtain (4) with $\lambda \simeq 2 / 7,[11]$. The rise of the multiplicity plateau is not proportional to $Y$ but to $\exp (\lambda Y)$.

Following $[12,13]$ we write, for $\eta \geq 0$,

$$
\left.\frac{d n}{d y}\right|^{s}=\frac{1}{e^{\frac{\eta-(1-\alpha) Y}{\delta}}+1}
$$

$\alpha$ and $\delta$ being free parameters. Finally, using the Jacobean $J$ of the $y \rightarrow \eta$ transformation, we construct $d n /\left.d \eta\right|_{p p}$,

$$
\left.\frac{d n}{d \eta}\right|_{p p}=\left.J \frac{d n}{d y}\right|_{p p}
$$

with $J=\frac{\cosh \eta}{\sqrt{k+\sinh ^{2} \eta}}, k=\frac{m^{2}+p_{T}^{2}}{p_{T}^{2}}$. and by assumption fix $k$ at the effective value 1.2. It corresponds to $m_{\pi} \simeq 0.14 \mathrm{GeV}$ and $\bar{p}_{\pi} \simeq 0.3 \mathrm{GeV}$ also in agreement with [14].

In Fig. 1 we show our fits to $d n_{c h} / d \eta$ for pp collisions (excluding single diffraction) from $53 \mathrm{GeV}$ to $1.8 \mathrm{TeV}$ [15], and at LHC energies [16]. The free parameters where fixed at $\lambda=0.23 \pm 0.005, \delta=0.61 \pm 0.15, \alpha=0.27 \pm 0.03$, $a=0.8 \pm 0.2$.

In Fig. 2 we show the evolution with $Y$ at different values of pseudorapidity, $\eta$. Evolution within the "plateau" is slower than evolution in the "fragmentation" region.

Making use of (6) and (7) it is easily seen what happens. If $\eta \geq 0$ is in the "plateau" region $d n / d \eta$ evolves as $e^{\lambda Y}$,

$$
\frac{d n}{d \eta}(\eta \leq(1-\alpha) Y, Y) \simeq e^{\lambda Y}
$$

If $|\eta|$ is in the "fragmentation" region $d n / d \eta$ evolves as $e^{\lambda Y+\frac{(1-\alpha)}{\delta} Y}$

$$
\frac{d n}{d \eta}(\eta \geq(1-\alpha) Y, Y) \sim e^{\lambda Y+\frac{(1-\alpha)}{\delta} Y} .
$$

For fixed $\eta$ with increasing $Y$, at some stage one falls in the situation (9), as seen in Fig. 2 . 


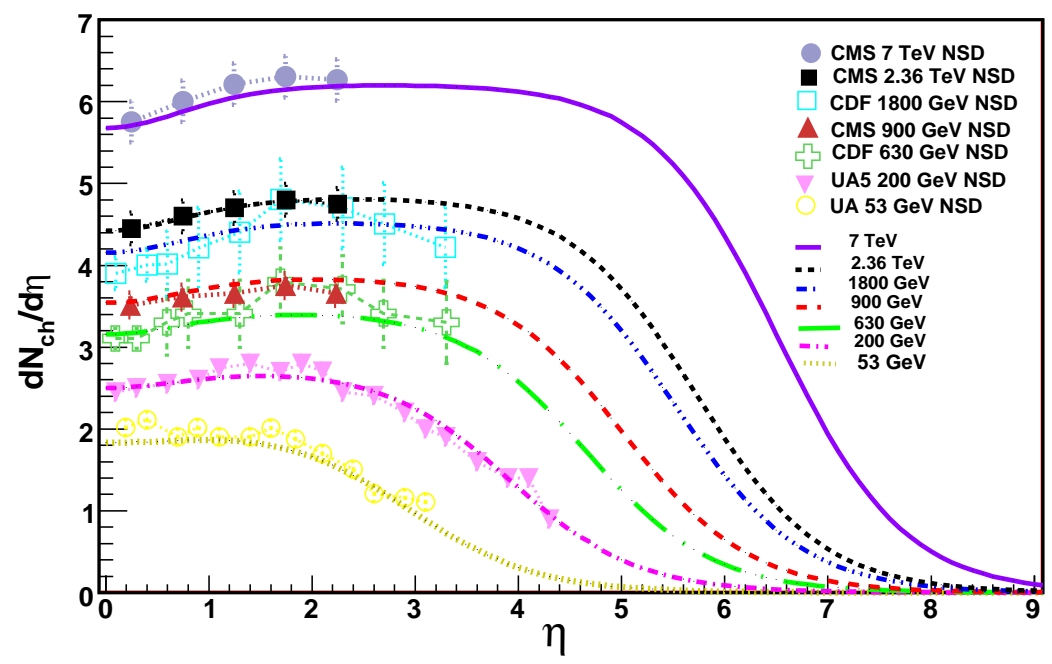

Figure 1: $d N_{c h} / d \eta$ at different $\sqrt{s}$. For data points see references [15],[16]. Data from UA5, $546 \mathrm{GeV}$ and UA1 at $540 \mathrm{GeV}$ being inconsistent, were not included. Data from P238, $630 \mathrm{GeV}$ are also not included. Note that the $\sqrt{s}=2.36 \mathrm{TeV}$ and $0.9 \mathrm{TeV}$ plotted is from CMS for clarity of the plot the ALICE results also follow in these range and they differ form the CMS data for less than 0.2 . 


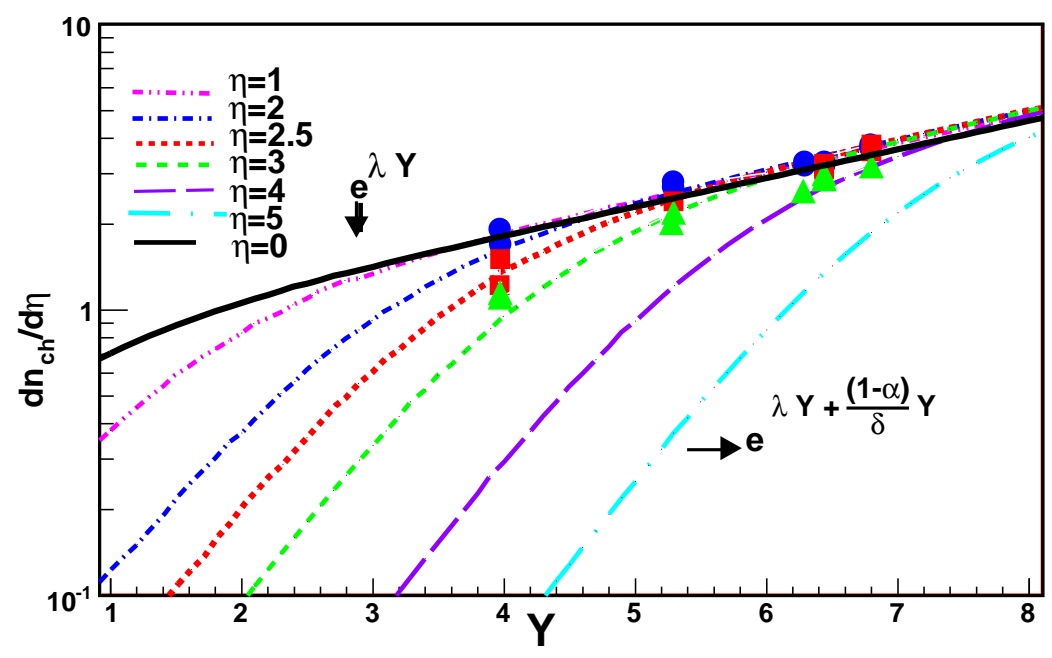

Figure 2: Comparison of the results from the evolution of the $d n_{c h} / d \eta$ with the beam rapidity from equation (7) for different fixed $\eta$ values. Blue circles are used for data at $\eta=2$, red squares are used for data at $\eta=2.5$, and green triangles are used for data at $\eta=3[15,16]$. 
Our main result is that if we start at a given $Y_{0}$ with $\left.\frac{d n}{d y}\right|_{\eta>0}<\left.\frac{d n}{d y}\right|_{\eta=0}$ after enough $Y$ evolution we end up with $\left.\left.\frac{d n}{d y}\right|_{\eta>0} \simeq \frac{d \eta}{d y}\right|_{\eta=0}$ (see Fig.2, where we have worked not with $\frac{d n}{d y}$ but with $\frac{d n}{d \eta}$ ). This means that a "plateau" proportional to $Y$ is developing.

Such "plateau" may be responsible for forward- backward long range rapidity correlations and the ridge phenomenon. It is well known, see for instance $[17,5]$, that in simple models the forward-backward correlation parameter $b$ depends on the "plateau" density. If we have a long distance true plateau, then $b$ is constant over a long distance. On the other hand the height of the ridge structure or the near ridge particles is also dependent on particle density. The same occurs in the frame of Color Glass Condensate model [18].

We discuss next the question of limiting fragmentation. For $\eta$ large, $\eta>(1-\alpha) Y$ we have

$$
\begin{array}{r}
\frac{d n}{d \eta} \simeq e^{\lambda Y+\frac{(1-\alpha)}{\delta} Y-\frac{\eta}{\delta}} \\
\simeq e^{\lambda(Y-\eta)+\frac{(1-\alpha)}{\delta} Y+\frac{(1-\lambda \delta)}{\delta}(-\eta)}
\end{array}
$$

The limiting fragmentation condition, i.e. the particle density being only a function of $Y-\eta$, is $1-\alpha=1-\lambda \delta$, or

$$
\alpha / \lambda=\delta .
$$

Experimentally $\alpha / \lambda>1$ and $\delta<1$ and including errors, $\alpha / \lambda=1.17 \pm 0.15$ and $\delta=.61 \pm 0.15$, we see that (12) is not satisfied.

To see what is expected we write (11) in the form

$$
\frac{d n}{d \eta} \sim e^{\lambda(Y-\eta)+\frac{(1-\alpha)}{\delta}(Y-\eta)+\left[\frac{(1-\lambda \delta)}{\delta}-\frac{(1-\alpha)}{\delta}\right](-\eta)}
$$

and $\frac{d n}{d \eta} \rightarrow 0$ as $\eta$ (or $Y$ ) goes to infinity. In this limit the distribution becomes close to a step function. It looks as if the fast particles in the fragmentation region disappear to feed up the front region in the plateau.

The arguments developed here for pp scattering, also apply to AA and pA collisions, to the extent that they have similar longitudinal structure. 


\section{Acknowledgments}

We thank J. G. Milhano for useful discussions. J. D. D. thanks the support of the FCT/Portugal project PPCDT/FIS/575682004. I. B. thanks the support of the FCT/Portugal SFRH/BD/51370/2011. I. B, and C. P. were supported by the project FPA 2008-01177 and FPA 2011-22776 of MICINN, the Spanish Consolider Ingenio 2010 program CPAN and Conselleria Educacion Xunta de Galicia.

\section{References}

1. L. V. Gribov, E. M. Levin and M. G. Ryskin, Phys. Rept. 100 (1983) 1; A. H. Mueller and J. w. Qiu, Nucl. Phys. B 268 (1986) 427.

2. L. D. McLerran and R. Venugopalan, Phys. Rev. D 49 (1994) 2233;

L. D. McLerran and R. Venugopalan, Phys. Rev. D 49 (1994) 3352;

L. D. McLerran and R. Venugopalan, Phys. Rev. D 50 (1994) 2225.

3. N. Armesto, M. A. Braun, E. G. Ferreiro and C. Pajares, Phys. Rev. Lett. 77 (1996) 3736; H. Satz, Nucl. Phys. A 642 (1998) 130 M. A. Braun and C. Pajares; Phys. Rev. Lett. 85 (2000) 4864.

4. J. Dias de Deus and R. Ugoccioni, Phys. Lett. B 491 (2000) 253; J. Dias de Deus and R. Ugoccioni, Phys. Lett. B 494 (2000) 53.

5. J. Dias de Deus and C. Pajares, Phys. Lett. B 695 (2011) 211.

6. C. Pajares and Yu. M. Shabelski, Relativistic Nuclear Interactions, URSS, Moscow (2007).

7. C. Pajares, Eur. Phys. J. C 43 (2005) 9; J. Dias de Deus and R. Ugoccioni, Eur. Phys. J. C 43 (2005) 249.

8. A. Rodrigues, R. Ugoccioni and J. Dias de Deus, Phys. Lett. B 458 (1999) 402.

9. M. A. Braun, F. Del Moral and C. Pajares, Phys. Rev. C 65 (2002) 024907.

10. A. M. Stasto, K. J. Golec-Biernat and J. Kwiecinski, Phys. Rev. Lett. 86 (2001) 596. 
11. J. Dias de Deus, M. C. Espirito Santo, M. Pimenta and C. Pajares, Phys. Rev. Lett. 96 (2006) 162001.

12. J. Dias de Deus and J. G. Milhano, Nucl. Phys. A 795 (2007) 98.

13. P. Brogueira, J. Dias de Deus and C. Pajares, Phys. Rev. C 75 (2007) 054908 .

14. G. Wolschin, Europhys. Lett. 95 (2011) 61001.

15. J. F. Grosse-Oetringhaus and K. Reygers, J. Phys. G 37 (2010) 083001.

16. K. Aamodt et al. [ALICE Collaboration], Eur. Phys. J. C 68 (2010) 89; K. Aamodt et al. [ALICE Collaboration], Eur. Phys. J. C 68 (2010) 345; V. Khachatryan et al. [CMS Collaboration], Phys. Rev. Lett. 105 (2010) 022002.

17. P. Brogueira, J. Dias de Deus and C. Pajares, Phys. Lett. B 675 (2009) 308.

18. N. Armesto, L. McLerran and C. Pajares, Nucl, Phys. A 78 (2007) 201 [hep-ph/0607345]; A. Dimitru, F. Gelis, L. McLerran, R. Venugopalan Nucl. Phys. A 81091 (2008). 